\title{
Bäcklund Transformations and Non-Abelian Nonlinear Evolution Equations: a Novel Bäcklund Chart ${ }^{\star}$
}

\author{
Sandra CARILLO ${ }^{\dagger^{1} \dagger^{2}}$, Mauro LO SCHIAVO ${ }^{\dagger^{1}}$ and Cornelia SCHIEBOLD ${ }^{\dagger^{3} \dagger^{4}}$ \\ $\dagger^{1}$ Dipartimento "Scienze di Base e Applicate per l'Ingegneria", Sapienza - Università di Roma, \\ 16, Via A. Scarpa, 00161 Rome, Italy \\ E-mail:sandra.carillo@sbai.uniroma1.it,mauro.loschiavo@sbai.uniroma1.it \\ URL: http://www.sbai.uniroma1.it/ sandra.carillo/ \\ URL: http://www.sbai.uniroma1.it/ mauro.loschiavo/ \\ $\dagger^{2}$ I.N.F.N. - Sez. Roma1, Gr. IV - Mathematical Methods in NonLinear Physics, Rome, Italy \\ $\dagger^{3}$ Department of Science Education and Mathematics, Mid Sweden University, \\ S-851 70 Sundsvall, Sweden \\ E-mail: Cornelia.Schiebold@miun.se \\ URL: http://www.miun.se/personal/corneliaschiebold \\ $\dagger^{4}$ Instytut Matematyki, Uniwersytet Jana Kochanowskiego w Kielcach, Poland
}

Received December 08, 2015, in final form August 24, 2016; Published online August 30, 2016

http://dx.doi.org/10.3842/SIGMA.2016.087

\begin{abstract}
Classes of third order non-Abelian evolution equations linked to that of Korteweg-de Vries-type are investigated and their connections represented in a non-commutative Bäcklund chart, generalizing results in [Fuchssteiner B., Carillo S., Phys. A 154 (1989), 467510]. The recursion operators are shown to be hereditary, thereby allowing the results to be extended to hierarchies. The present study is devoted to operator nonlinear evolution equations: general results are presented. The implied applications referring to finite-dimensional cases will be considered separately.
\end{abstract}

Dedicated to Sergio Benenti on his 70th birthday

\section{Introduction}

Bäcklund transformations and their associated nonlinear superposition principles, known as permutability theorems, play a central role in modern soliton theory as described in [29, 48, 49], see also [46] for further applications. The importance of the combination of gauge and reciprocal transformations to link hierarchies of integrable systems is well-established (see, e.g., [42] and literature cited therein). Here, the concern is with the application of Bäcklund transformations to connect various non-Abelian equations to a canonical $\mathrm{KdV}$-type equation and to construct their recursion operators. Hierarchies of such nonlinear equations are considered wherein the unknown is an operator on a Banach space. Such operator equations were originally introduced by Marchenko [39] and further investigated and developed, in the framework of Banach space geometry, in $[1,15,16]$.

The results comprised in this article represent the continuation of the study in $[9,11,12$, 13, 14]. In particular, in [11, 13] the focus is on the operator potential Korteweg-de Vries, Korteweg-de Vries and modified Korteweg-de Vries equations, their connections via Bäcklund transformations and the construction of the recursion operators they admit.

\footnotetext{
* This paper is a contribution to the Special Issue on Analytical Mechanics and Differential Geometry in honour of Sergio Benenti. The full collection is available at http://www.emis.de/journals/SIGMA/Benenti.html
} 
One of the main advantages of connecting non-Abelian equations via Bäcklund transformations is that solutions can be transferred from one equation to another. In [11] operator-valued solutions (which can be interpreted as operator analogs of solitons) to the pKdV, KdV and mKdV hierarchies are constructed. In [13] suitable projection techniques are exploited to derive solution formulae to the corresponding scalar and matrix hierarchies. Note that the study of non-Abelian nonlinear evolution equations found its original interest in the case of matrix equations $[6,36]$. The results in the present article are valid for operator-valued functions. This level of generality permits to construct solutions to scalar and matrix equations that can be viewed as countable superposition of solitons, see also $[50,53]$ for the connection between countable nonlinear superposition and Banach space geometry, and [51, 52] for further applications.

In [10], the term Bäcklund chart was introduced to indicate the net of Bäcklund transformations connecting different evolution equations. In [23] a wide Bäcklund chart which includes scalar 3rd order Abelian evolution equations is constructed. It connects, further to the $\mathrm{KdV}$ and the modified $\mathrm{KdV}$ equations, in particular the $\mathrm{KdV}$ singularity manifold equation, also known as as UrKdV or Schwarz-KdV [18, 55, 57, 58], and the KdV interacting soliton equation [22]. The connections among the equations in this Abelian Bäcklund chart are applied to find, or recover, the recursion operators admitted by all these nonlinear evolution equations. Moreover, other structural properties such as the Hamiltonian, and bi-Hamiltonian, structure of these equations are also obtained via the Bäcklund chart [23]. The present study is concerned about the extension of the Bäcklund chart in [11] to obtain the non-Abelian analog of the links established in the scalar case [23]. Precisely, in addition to the $\mathrm{pKdV}, \mathrm{KdV}$ and $\mathrm{mKdV}$ equations already connected in [11], a different version of the non-Abelian modified KdV and two non-Abelian equations, respectively, analogs of the KdV singularity manifold and of the KdV interacting soliton equation, are all linked together via Bäcklund transformations. New, in the present Bäcklund chart, are the inclusion of a second modified KdV equation, denoted as amKdV, for alternative $\mathrm{mKdV}$ equation [34, 44], new are also the non-Abelian $\mathrm{KdV}$ singularity manifold equation and the non-Abelian $\mathrm{KdV}$ interacting soliton equation.

All the non-Abelian nonlinear evolution equations in the Bäcklund chart admit a recursion operator. The recursion operators of some of them, such as the pKdV, KdV and mKdV [11, 44, 54] are known. We recover, via the established connections, the recursion operators admitted by the non-Abelian amKdV, given in [44]. Then, the recursion operators of the non-Abelian KdV singularity manifold and of the KdV interacting soliton equations, both new, are constructed. Furthermore, the hereditariness of all the obtained recursion operators is proved combining links via Bäcklund transformations [19, 20], with the hereditariness of non-Abelian KdV recursion operator [54]. Finally, since all the nonlinear third order non-Abelian evolution equations admit hereditary recursion operators, according to [19, 20], all the links in the Bäcklund chart can be extended to the corresponding whole hierarchies.

The material is organized as follows. The opening Section 2 is devoted to the Bäcklund charts connecting nonlinear evolution equations which generalize, to the operator level, the $\mathrm{pKdV}, \mathrm{KdV}$ and $\mathrm{mKdV}$ equations $[11,13]$. Sections 3 and 4 are devoted to the construction of a novel Bäcklund chart. In Section 3, the Bäcklund chart is extended to incorporate also the non-Abelian amKdV equation; its recursion operator is constructed from the known recursion operators of the mKdV equations; finally, the connection between the two different non-Abelian modified $\mathrm{KdV}$ equations is provided.

In Section 4, the novel Bäcklund chart is further enlarged. Then, the recursion operators of the $\mathrm{KdV}$ interacting soliton and of the KdV singularity manifold equations are constructed. In addition, in Section 4, a Möbius type invariance exhibited by the non-Abelian KdV singularity manifold equation is established.

The subsequent Section 5 is devoted to the proof of hereditariness of all the recursion operators in the previous sections. The hereditariness of all the recursion operators in the Bäcklund chart 
guarantees that the links can be transferred to whole hierarchies, relating their corresponding members. In the closing Section 6, further to some remarks on the interest of the present study, open problems and perspectives suggested by our new results are briefly outlined. The article is complemented with an Appendix where some needed definitions, such as the definition of Bäcklund transformation [19], adopted throughout, as well as known results obtained in the case of Abelian nonlinear evolution equations [23] are comprised.

\section{Non-Abelian Bäcklund charts}

In [11], the recursion operators admitted by the non-Abelian potential $\mathrm{KdV}, \mathrm{KdV}$ and modified $\mathrm{KdV}$ equations were shown to be related by Bäcklund transformations. Consider first the operator potential KdV equation $(\mathrm{pKdV})$

$$
W_{t}=W_{x x x}+3 W_{x}^{2}
$$

where the unknown $W$ is a function whose values are bounded linear operators on some Banach space $^{1}$. It admits the recursion operator

$$
\hat{\Psi}(W)=D^{2}+A_{W_{x}}+D^{-1} A_{W_{x}} D+D^{-1} C_{W_{x}} D^{-1} C_{W_{x}},
$$

where $D$ denotes the derivative with respect to $x$, and $C_{T}, A_{T}$ denote the commutator and anti-commutator with respect to $T$, namely,

$$
C_{T} S:=[T, S] \equiv T S-S T, \quad A_{T} S:=\{T, S\} \equiv T S+S T .
$$

For earlier occurrences of recursion operators in the non-Abelian setting we refer to [5, 25, 32, 44]. Consider next the operator Korteweg-de Vries equation (KdV)

$$
U_{t}=U_{x x x}+3\left\{U, U_{x}\right\}
$$

which admits the recursion operator

$$
\Phi(U)=D^{2}+2 A_{U}+A_{U_{x}} D^{-1}+C_{U} D^{-1} C_{U} D^{-1} .
$$

The recursion operators (2.2) and (2.4) are linked via the Bäcklund transformation

$$
B_{1}: U-W_{x}=0 .
$$

In fact, the transformation operator is $\Pi_{B_{1}}=D^{-1}$, and $\hat{\Psi}(W)=D^{-1} \Phi(U) D$. Hence, the non-Abelian pKdV (2.1) and $\mathrm{KdV}(2.3)$ equations can also be written as

$$
W_{t}=\hat{\Phi}(W) W_{x} \quad \text { and } \quad U_{t}=\Phi(U) U_{x}
$$

Finally, the $\mathrm{KdV}$ equation (2.3) is related to the operator modified $\mathrm{KdV}$ equation (mKdV)

$$
V_{t}=V_{x x x}-3\left\{V^{2}, V_{x}\right\}
$$

via the Miura transformation

$$
M: U+V_{x}+V^{2}=0 .
$$

\footnotetext{
${ }^{1}$ Here capital letters are used to emphasize that the unknown is an operator acting on a Banach space.
} 
Hence, the Miura transformation (2.6) allows to obtain the recursion operator $\Psi(V)$ of the $\mathrm{mKdV}(2.5)$ from the recursion operator $\Phi(U)$ of the $\mathrm{KdV}(2.4)$ via

$$
\Psi(V)=\Pi_{M} \Phi(U) \Pi_{M}^{-1}, \quad \text { where } \quad \Pi_{M}=-\left(D+A_{V}\right)^{-1} .
$$

The latter implies

$$
\Psi(V)=\left(D-C_{V} D^{-1} C_{V}\right)\left(D-A_{V} D^{-1} A_{V}\right)
$$

(see [11] for details), which can be equivalently written as

$$
\Psi(V)=\left(D-C_{V}\right) D^{-1}\left(D+C_{V}\right)\left(D-A_{V}\right) D^{-1}\left(D+A_{V}\right) .
$$

The following Bäcklund chart summarizes the links among the non-Abelian pKdV (2.1), KdV (2.3), and $\mathrm{mKdV}(2.5)$ equations:

$$
W_{t}=W_{x x x}+3 W_{x}^{2} \frac{B_{1}}{-} U_{t}=U_{x x x}+3\left\{U, U_{x}\right\} \stackrel{M}{-} V_{t}=V_{x x x}-3\left\{V^{2}, V_{x}\right\} .
$$

When the respective recursion operators are applied to the above equations iteratively, the Bäcklund chart can be extended to the corresponding hierarchies, and the connections can be summarized in

$$
W_{t}=[\hat{\Phi}(W)]^{n} W_{x} \frac{B_{1}}{U_{t}=[\Phi(U)]^{n} U_{x}} \stackrel{M}{V_{t}=[\Psi(V)]^{n} V_{x} .}
$$

Note that (2.8) is the natural non-Abelian counterpart of the corresponding part of the Bäcklund chart (A.4) introduced in [23].

\section{On the non-Abelian mKdV equations}

A distinguished feature of the Bäcklund chart studied in this article is that it proceeds via two versions of the non-Abelian $\mathrm{mKdV}$ equation. The link between those versions is considered in this section. The alternative non-Abelian mKdV equation (amKdV)

$$
\widetilde{V}_{t}=\widetilde{V}_{x x x}+3\left[\widetilde{V}, \widetilde{V}_{x x}\right]-6 \widetilde{V} \widetilde{V}_{x} \widetilde{V}
$$

was first described in [34], where the Lax pair formulation and the inverse scattering problem were studied. In contrast to the non-Abelian mKdV (2.5) studied in the previous section, (3.1) does not admit a Miura transformation [44]. Note also that, in the matrix case, (2.5) is invariant under both $V \rightarrow V^{*}$ and $V \rightarrow-V$, whereas (3.1) is only invariant under $\widetilde{V} \rightarrow-\widetilde{V}^{*}\left(V^{*}\right.$ denoting the transpose of $V$ ).

As already pointed out in [37], these two versions of the $\mathrm{mKdV}$ equation are linked by the gauge transformation $\widetilde{V}=G^{-1} V G$ with $G_{x}=V G$. Consider the Bäcklund transformations

$$
\begin{aligned}
& B_{2}: G_{x}-V G=0, \\
& B_{3}: G_{x}-G \widetilde{V}=0 .
\end{aligned}
$$

Obviously, subsequent application of these Bäcklund transformations links (2.5) to (3.1).

The recursion operator $\widetilde{\Psi}(\widetilde{V})$ of $(3.1)$ is

$$
\widetilde{\Psi}(\widetilde{V})=\left(D+2 C_{\widetilde{V}}\right)\left(D-2 R_{\widetilde{V}}\right)\left(D+C_{\widetilde{V}}\right)^{-1}\left(D+2 L_{\widetilde{V}}\right) D\left(D+C_{\widetilde{V}}\right)^{-1}
$$

It was first given in [32], where it was derived using Lax representation. Here we use the Bäcklund link between the $\mathrm{mKdV}$ and the amKdV equation to give an alternative derivation together with a more conceptual formulation of the operator itself.

To this end, we introduce the derivation

$$
\mathbb{D}:=D+C_{\widetilde{V}} .
$$


Theorem 3.1. On use of the derivation (3.5), the recursion operator (3.4) of the amKdV equation can be written as

$$
\widetilde{\Psi}(\widetilde{V})=\left(\mathbb{D}+C_{\widetilde{V}}\right)\left(\mathbb{D}-A_{\widetilde{V}}\right) \mathbb{D}^{-1}\left(\mathbb{D}+A_{\widetilde{V}}\right)\left(\mathbb{D}-C_{\widetilde{V}}\right) \mathbb{D}^{-1} .
$$

Remark 3.2. Recall that two operators $T$ and $S$ are called related if they are of the form $T=A B$ and $S=B A$. In [11], relatedness of the non-Abelian $\mathrm{KdV}$ and $\mathrm{mKdV}$ recursion operators on the image of the Miura transform was exploited to derive solutions of the nonAbelian mKdV hierarchy from solutions of the non-Abelian KdV hierarchy.

Here we observe that $\Psi(V)$ and $\widetilde{\Psi}(\widetilde{V})$ are related in a generalized sense: If $P(D, V)=$ $\left(D-C_{V}\right) D^{-1}$ and $Q(D, V)=\left(D+C_{V}\right)\left(D-A_{V}\right) D^{-1}\left(D+A_{V}\right)$, then

$$
\Psi(V)=P(D, V) Q(D, V), \quad \widetilde{\Psi}(\widetilde{V})=Q(\mathbb{D}, \widetilde{V}) P(\mathbb{D}, \widetilde{V}) .
$$

Before proving Theorem 3.1, we observe some crucial properties of the derivation (3.5).

Proposition 3.3. Let $V$ and $\widetilde{V}$ be related to each other via the Bäcklund transformations $B_{2}$ and $B_{3}$ with some intermediate invertible function $G$. Then the derivation (3.5) is the result of conjugation of $D$ with $K_{G}^{-1}$, i.e.,

$$
\mathbb{D}=K_{G} D K_{G}^{-1},
$$

where $K_{G}$ denotes the conjugation operator, $K_{G} T=G^{-1} T G$.

Moreover, it holds:

a) $K_{G}\left(D \pm C_{V}\right) K_{G}^{-1}=\mathbb{D} \pm C_{\widetilde{V}}$,

b) $K_{G}\left(D \pm A_{V}\right) K_{G}^{-1}=\mathbb{D} \pm A_{\widetilde{V}}$.

Proof. As an example, we verify $K_{G} D K_{G}^{-1}=\mathbb{D}$ and $K_{G} C_{V} K_{G}^{-1}=C_{\widetilde{V}}$.

Indeed,

$$
\begin{aligned}
K_{G} D K_{G}^{-1} T & =G^{-1}\left(G T G^{-1}\right)_{x} G=G^{-1}\left(G_{x} T G^{-1}+G T_{x} G^{-1}-G T G^{-1} G_{x} G^{-1}\right) G \\
& =G^{-1} G_{x} T+T_{x}-T G^{-1} G_{x}=\widetilde{V} T+T_{x}-T \widetilde{V}=\left(D+C_{\widetilde{V}}\right) T=\mathbb{D} T .
\end{aligned}
$$

Analogously,

$$
\begin{aligned}
K_{G} C_{V} K_{G}^{-1} T & =G^{-1}\left[V, G T G^{-1}\right] G=G^{-1}\left(V G T G^{-1}-G T G^{-1} V\right) G \\
& =G^{-1} V G T-T G^{-1} V G=\left[G^{-1} V G, T\right]=[\widetilde{V}, T]=C_{\widetilde{V}} T .
\end{aligned}
$$

Proof of Theorem 3.1. On use of the Bäcklund transformations $B_{2}, B_{3}$, the amKdV recursion operator $\widetilde{\Psi}(\widetilde{V})$ is related to the mKdV recursion operator $\Psi(V)$ via

$$
\widetilde{\Psi}(\widetilde{V})=\Pi \Psi(V) \Pi^{-1}
$$

with $\Pi=\Pi_{3} \Pi_{2}$, where

$$
\Pi_{2}=-\left(B_{2}\right)_{G}^{-1}\left(B_{2}\right)_{V}, \quad \Pi_{3}=-\left(B_{3}\right)_{\widetilde{V}}^{-1}\left(B_{3}\right)_{G} .
$$

Let $L_{V}$ and $R_{V}$ denote left and right multiplication by $V$, then $C_{V} T=\left(L_{V}-R_{V}\right) T$ and $A_{V} T=\left(L_{V}+R_{V}\right) T$. Now it is straightforward to verify $\left(B_{2}\right)_{G}=\left(D-L_{V}\right),\left(B_{2}\right)_{V}=-R_{G}$. This leads to the transformation operator $\Pi_{2}=\left(D-L_{V}\right)^{-1} R_{G}$. Similarly, $\left(B_{3}\right)_{G}=\left(D-R_{\widetilde{V}}\right)$, $\left(B_{3}\right)_{\widetilde{V}}=-L_{G}$ leading to $\Pi_{3}=L_{G^{-1}}\left(D-R_{\widetilde{V}}\right)$. Hence,

$$
\Pi=L_{G^{-1}}\left(D-R_{\widetilde{V}}\right)\left(D-L_{V}\right)^{-1} R_{G} .
$$


Next, using the identities $\left(D-L_{V}\right) R_{G}=R_{G}\left(D-C_{V}\right)$ and $\left(D-R_{\widetilde{V}}\right) R_{G}=R_{G} D$, which are straightforward to check, it follows

$$
\begin{aligned}
\Pi & =L_{G^{-1}}\left(D-R_{\widetilde{V}}\right)\left(\left(D-L_{V}\right)^{-1} R_{G}\right)=L_{G^{-1}}\left(\left(D-R_{\widetilde{V}}\right) R_{G}\right)\left(D-C_{V}\right)^{-1} \\
& =L_{G^{-1}} R_{G} D\left(D-C_{V}\right)^{-1}=K_{G} D\left(D-C_{V}\right)^{-1} .
\end{aligned}
$$

Hence, the amKdV recursion operator $\widetilde{\Psi}(\widetilde{V})$ is obtained from the recursion operator $\Psi(V)$ of the $\mathrm{mKdV}$ equation as stated in (2.7) via

$$
\begin{aligned}
\widetilde{\Psi}(\widetilde{V}) \stackrel{(3.6),(3.7)}{=} & \left(K_{G} D\left(D-C_{V}\right)^{-1}\right)\left(D-C_{V}\right) D^{-1}\left(D+C_{V}\right)\left(D-A_{V}\right) \\
& \times D^{-1}\left(D+A_{V}\right)\left(\left(D-C_{V}\right) D^{-1} K_{G^{-1}}\right) \\
= & K_{G^{-1}}\left(D+C_{V}\right)\left(D-A_{V}\right) D^{-1}\left(D+A_{V}\right)\left(D-C_{V}\right) D^{-1} K_{G} \\
= & \left(\mathbb{D}+C_{\widetilde{V}}\right)\left(\mathbb{D}-A_{\widetilde{V}}\right) \mathbb{D}^{-1}\left(\mathbb{D}+A_{\widetilde{V}}\right)\left(\mathbb{D}-C_{\widetilde{V}}\right) \mathbb{D}^{-1},
\end{aligned}
$$

where the last step requires Proposition 3.3.

To conclude this section, for the reader's convenience we give a direct verification that the $\mathrm{mKdV}$ and the amKdV equation are related via $\widetilde{V}=G^{-1} G_{x}$ and $V=G_{x} G^{-1}$. More precisely, we show

a) $V_{t}=\Psi(V) V_{x} \Longleftrightarrow \widetilde{V}_{t}=\widetilde{\Psi}(\widetilde{V}) \widetilde{V}_{x}$,

b) $\widetilde{\Psi}(\widetilde{V}) \widetilde{V}_{x}$ constitutes the right-hand side of (3.1).

To prove a), recall that $\widetilde{\Psi}(\widetilde{V})=\Pi \Psi(V) \Pi^{-1}$ where $\Pi=K_{G} D\left(D-C_{V}\right)^{-1}$, compare (3.6), (3.7) in the proof of Theorem 3.1. Noting that $\widetilde{V}=K_{G} V$ implies $\widetilde{V}_{x}=K_{G} V_{x}$, since $\left(G^{-1} G_{x}\right)_{x}=$ $G^{-1}\left(G_{x} G^{-1}\right)_{x} G$ (observe that $\widetilde{V}_{x x}=K_{G} V_{x x}$ does not hold). Hence

$$
\Pi^{-1} \widetilde{V}_{x} \stackrel{(3.7)}{=}\left(D-C_{V}\right) D^{-1} K_{G}^{-1} \widetilde{V}_{x}=\left(D-C_{V}\right) D^{-1} V_{x}=\left(D-C_{V}\right) V=V_{x} .
$$

Furthermore, $\widetilde{V}_{t}=\left(G^{-1} G_{x}\right)_{t}=G^{-1} G_{x t}-G^{-1} G_{t} G^{-1} G_{x}=G^{-1}\left(G_{x t} G^{-1}-G_{t} G^{-1} G_{x} G^{-1}\right) G=$ $K_{G} D\left(G_{t} G^{-1}\right)$. This shows

$$
\Pi^{-1} \widetilde{V}_{t}=\left(D-C_{V}\right) D^{-1} K_{G}^{-1} \widetilde{V}_{t}=\left(D-C_{V}\right)\left(G_{t} G^{-1}\right)=V_{t} .
$$

Now a) follows from $\widetilde{\Psi}(\widetilde{V}) \widetilde{V}_{x}=\Pi \Psi(V) \Pi^{-1} \widetilde{V}_{x} \stackrel{(3.8)}{=} \Pi \Psi(V) V_{x}=\Pi V_{t} \stackrel{(3.9)}{=} \widetilde{V}_{t}$.

To prove b), note first that $\widetilde{V}_{x}=D \widetilde{V}=\left(D+C_{\widetilde{V}}\right) \widetilde{V}=\mathbb{D} \widetilde{V}$. Similarly, $\left(\mathbb{D}-C_{\widetilde{V}}\right) \widetilde{V}=\mathbb{D} \widetilde{V}$, and then $\left(\mathbb{D}+A_{\widetilde{V}}\right) \mathbb{D} \widetilde{V}=\mathbb{D}^{2} \widetilde{V}+\{\widetilde{V}, \mathbb{D} \widetilde{V}\}=\mathbb{D}\left(\widetilde{V}^{2}+\mathbb{D} \widetilde{V}\right)$. Using Theorem 3.1, we hence get

$$
\begin{aligned}
\widetilde{\Psi}(\widetilde{V}) \widetilde{V}_{x} & =\left(\mathbb{D}+C_{\widetilde{V}}\right)\left(\mathbb{D}-A_{\widetilde{V}}\right) \mathbb{D}^{-1}\left(\mathbb{D}+A_{\widetilde{V}}\right)\left(\mathbb{D}-C_{\widetilde{V}}\right) \mathbb{D}^{-1} \mathbb{D} \widetilde{V} \\
& =\left(\mathbb{D}+C_{\widetilde{V}}\right)\left(\mathbb{D}-A_{\widetilde{V}}\right) \mathbb{D}^{-1}\left(\mathbb{D}+A_{\widetilde{V}}\right) \mathbb{D} \widetilde{V}=\left(\mathbb{D}+C_{\widetilde{V}}\right)\left(\mathbb{D}-A_{\widetilde{V}}\right)\left(\mathbb{D} \widetilde{V}+\widetilde{V}^{2}\right) \\
& =\left(\mathbb{D}+C_{\widetilde{V}}\right)\left(\mathbb{D}^{2} \widetilde{V}-2 \widetilde{V}^{3}\right)=\mathbb{D}^{3} \widetilde{V}+\left[\widetilde{V}, \mathbb{D}^{2} \widetilde{V}\right]-2 \mathbb{D}\left(\widetilde{V}^{3}\right) .
\end{aligned}
$$

Finally, evaluation of $\mathbb{D}=D+C_{\widetilde{V}}$ gives the right-hand side of the amKdV equation.

\section{A new non-Abelian Bäcklund chart}

In the last two sections a non-Abelian generalization of the Bäcklund chart linking $\mathrm{pKdV}, \mathrm{KdV}$ and $m K d V$ equations was presented, and extended to include the amKdV equation (3.1). The aim of the present section is a further extension to also include non-Abelian analogues of the $\mathrm{KdV}$ singularity manifold equation and the KdV interacting soliton equation. To the best of the authors' knowledge, the non-Abelian equations presented in this sections are new. Together with the links presented in the previous section, the resulting non-Abelian Bäcklund chart ${ }^{2}$ we

\footnotetext{
${ }^{2}$ To facilitate the comparison with the Abelian case, a brief overview of the results in [23] is provided in the Appendix.
} 
obtain can be summarized as follows:

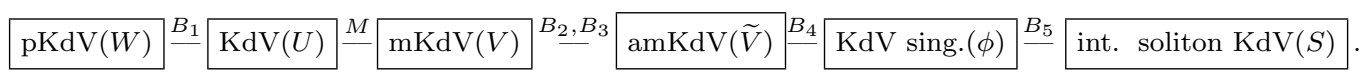

Moreover, the corresponding recursion operators are derived. Note that this chart extends to the respective hierarchies. In conclusion, it is shown that all recursion operators are hereditary. Finally, it is well-known that the KdV singularity manifold equation is invariant under the full Möbius group, and it is shown that a generalized property holds for its non-Abelian counterpart.

To begin with, we introduce a non-Abelian analogue of the KdV singularity manifold equation (KdV sing.)

$$
\phi_{t}=\phi_{x}\{\phi ; x\}
$$

where $\{\phi ; x\}$ denotes the following non-Abelian version of the Schwarzian derivative

$$
\{\phi ; x\}:=\left(\phi_{x}^{-1} \phi_{x x}\right)_{x}-\frac{1}{2}\left(\phi_{x}^{-1} \phi_{x x}\right)^{2} .
$$

\section{Remark 4.1.}

a) To the best of the authors' knowledge, equation (4.2) is new. For earlier non-Abelian versions of (4.2) we refer to [56], see also [2]. The difference to our approach is the non-Abelian interpretation of terms of the form $1 / u_{x}^{2}$.

b) In the scalar case there is a very satisfying group theoretic explanation of the relation between the KdV sing. (also called UrKdV), mKdV and KdV equations [58], see also [18, 55]. It seems to be an interesting problem to find an appropriate extension of these ideas to the non-Abelian setting. Departing from the present results, a first major difficulty is that our link between (2.5) and (3.1) (which reduce to the same scalar equation) is not realized by an explicit mapping $V=F(\widetilde{V})$.

The KdV sing. equation (4.2) exhibits invariances analogous to its scalar counterpart. Note that the following theorem implies invariance under the full Möbius group in the scalar case.

Theorem 4.2. Let $A, B, C, D$ be constant operators. Then the non-Abelian KdV singularity manifold equation (4.2) is invariant under the transformations

$$
\phi \mapsto(\phi A+B)^{-1}(\phi C+D), \quad \phi \mapsto(C \phi+D)(A \phi+B)^{-1},
$$

provided that $A$ and $D-B A^{-1} C$ are invertible.

In other words, if $\phi$ is an invertible solution of $(4.2)$, then also $(\phi A+B)^{-1}(\phi C+D)$ and $(C \phi+D)(A \phi+B)^{-1}$ are solutions of (4.2).

Remark 4.3. The condition that $D-B A^{-1} C$ is invertible can be understood as a generalization of the scalar condition $a d-b c \neq 0$ in the case $a \neq 0$. Note however, that this is not the only possibility to guarantee the invariances of Theorem 4.2. For example, the assumption that $B$ and $D-A B^{-1} C$ are invertible is also sufficient.

The main ingredient of the proof of Theorem 4.2 is the following observation.

Proposition 4.4. The non-Abelian KdV singularity manifold equation (4.2) is invariant under the transformation $\phi \mapsto \phi^{-1}$. 
Proof. To start with we calculate the Schwarzian derivative of $\psi:=\phi^{-1}$.

Since $\psi_{x}=-\phi^{-1} \phi_{x} \phi^{-1}$, we get $\psi_{x x}=-\phi^{-1} \phi_{x x} \phi^{-1}+2 \phi^{-1} \phi_{x} \phi^{-1} \phi_{x} \phi^{-1}$. Hence

$$
\psi_{x}^{-1} \psi_{x x}=\phi \phi_{x}^{-1} \phi_{x x} \phi^{-1}-2 \phi_{x} \phi^{-1},
$$

and therefore

$$
\begin{aligned}
\{\psi ; x\}= & \left(\psi_{x}^{-1} \psi_{x x}\right)_{x}-\frac{1}{2}\left(\psi_{x}^{-1} \psi_{x x}\right)^{2} \\
& =\phi_{x x} \phi^{-1}+\phi\left(\phi_{x}^{-1} \phi_{x x}\right)_{x} \phi^{-1}-\phi \phi_{x}^{-1} \phi_{x x} \phi^{-1} \phi_{x} \phi^{-1}-2 \phi_{x x} \phi^{-1}+2 \phi_{x} \phi^{-1} \phi_{x} \phi^{-1} \\
& -\frac{1}{2} \phi\left(\phi_{x}^{-1} \phi_{x x}\right)^{2} \phi^{-1}+\phi \phi_{x}^{-1} \phi_{x x} \phi^{-1} \phi_{x} \phi^{-1}+\phi_{x x} \phi^{-1}-2 \phi_{x} \phi^{-1} \phi_{x} \phi^{-1} .
\end{aligned}
$$

All terms with exception of the boxed ones cancel out, hence $\{\psi ; x\}=\phi\{\phi, x\} \phi^{-1}$. As a result,

$$
\psi_{t}=-\phi^{-1} \phi_{t} \phi^{-1} \stackrel{(4.2)}{=}-\phi^{-1}\left(\phi_{x}\{\phi ; x\}\right) \phi^{-1}=\left(-\phi^{-1} \phi_{x} \phi^{-1}\right)\left(\phi\{\phi ; x\} \phi^{-1}\right)=\psi_{x}\{\psi ; x\},
$$

which completes the proof.

The following invariance is straightforward to verify.

Proposition 4.5. The non-Abelian KdV singularity manifold equation (4.2) is invariant under the transformation $\phi \mapsto A \phi B+C$ where $A, B, C$ are constant operators, provided that $A$ and $B$ are invertible.

Proof of Theorem 4.2. Let $\phi$ be a solution of (4.2). By Proposition 4.5, also $\phi A+B$ solves (4.2) since $A$ is invertible. Then, by Proposition 4.4, the inverse $\psi=(\phi A+B)^{-1}$ is a solution of (4.2). Applying Proposition 4.5, $\psi\left(D-B A^{-1} C\right)+A^{-1} C=(\phi A+B)^{-1}(D-$ $\left.B A^{-1} C+(\phi A+B) A^{-1} C\right)=(\phi A+B)^{-1}(\phi C+D)$ is a solution of (4.2).

Finally, we introduce the non-Abelian KdV interacting soliton equation (int. soliton KdV)

$$
S_{t}=\left(S\left(\left(S^{-1} S_{x}\right)_{x}-\frac{1}{2}\left(S^{-1} S_{x}\right)^{2}\right)\right)_{x} .
$$

Again, to the best of the authors' knowledge, this equation is new.

Analogously as in Section 3, it can be verified that the amKdV (3.1), KdV sing. (4.2) and int. soliton $\mathrm{KdV}$ (4.3) equations are related by the Bäcklund transformations

$$
\begin{aligned}
& B_{4}: 2 \phi_{x} \widetilde{V}-\phi_{x x}=0, \\
& B_{5}: \phi_{x}-S=0 .
\end{aligned}
$$

Remark 4.6. Note that the counterpart of the transformation $\widetilde{V}=\frac{1}{2} \phi_{x}^{-1} \phi_{x x}$ from (4.4), namely, $\widehat{V}=-\frac{1}{2} \phi_{x x} \phi_{x}^{-1}$, also maps solutions $\phi$ of the $\mathrm{KdV}$ sing. equation (4.2) to solutions $\widehat{V}$ of the amKdV equation (3.1).

Both the recursion operators $\Upsilon(\phi)$ of (4.2) as well as $\chi(S)$ of (4.3) can be constructed on use of the Bäcklund links (4.4), (4.5).

Theorem 4.7. Define $N(T)=\frac{1}{2} T^{-1} T_{x}$. Then the recursion operators $\Upsilon(\phi)$ of $K d V$ sing. (4.2) and $\chi(S)$ of (4.3) are given by

$$
\begin{aligned}
& \Upsilon(\phi)=L_{\phi_{x}} \mathbb{D}^{-1}\left(\mathbb{D}-A_{N\left(\phi_{x}\right)}\right)\left(\mathbb{D}-C_{N\left(\phi_{x}\right)} \mathbb{D}^{-1} C_{N\left(\phi_{x}\right)}\right)\left(\mathbb{D}+A_{N\left(\phi_{x}\right)}\right) L_{\phi_{x}^{-1}}, \\
& \chi(S)=L_{S}\left(\mathbb{D}-A_{N(S)} \mathbb{D}^{-1} A_{N(S)}\right)\left(\mathbb{D}-C_{N(S)} \mathbb{D}^{-1} C_{N(S)}\right) L_{S}^{-1} .
\end{aligned}
$$

Note that $\mathbb{D}=D+C_{N\left(\phi_{x}\right)}=D+C_{N(S)}$. 
Proof. Indeed, from the Bäcklund links we have that the recursion operators satisfy

$$
\begin{array}{ll}
\Upsilon(\phi)=\Pi_{4} \widetilde{\Psi}(\widetilde{V}) \Pi_{4}^{-1}, & \Pi_{4}=-\left(B_{4}\right)_{\phi}^{-1}\left(B_{4}\right)_{\widetilde{V}}, \\
\chi(S)=\Pi_{5} \Upsilon(\phi) \Pi_{5}^{-1}, & \Pi_{5}=-\left(B_{5}\right)_{S}^{-1}\left(B_{5}\right)_{\phi} .
\end{array}
$$

It is straightforward to calculate $\left(B_{4}\right)_{\widetilde{V}}=2 L_{\phi_{x}},\left(B_{4}\right)_{\phi}=-\left(D-2 R_{\widetilde{V}}\right) D$. Therefore, we get

$$
\Pi_{4}=2 D^{-1}\left(D-2 R_{\widetilde{V}}\right)^{-1} L_{\phi_{x}} .
$$

Similarly $\left(B_{5}\right)_{S}=-I,\left(B_{5}\right)_{\phi}=D$, and hence $\Pi_{5}=D$. Consequently,

$$
\Pi_{5} \Pi_{4}=2\left(D-2 R_{\widetilde{V}}\right)^{-1} L_{\phi_{x}}=2\left(\mathbb{D}-A_{\widetilde{V}}\right)^{-1} L_{S} .
$$

Using the identity $\left(\mathbb{D}-A_{\widetilde{V}}\right) L_{S}=L_{S}\left(\mathbb{D}+C_{\widetilde{V}}\right)$, which can be checked directly using only the product rule and $\widetilde{V}=\frac{1}{2} S^{-1} S_{x}$, we find

$$
\Pi_{5} \Pi_{4}=2 L_{S}\left(\mathbb{D}+C_{\widetilde{V}}\right)^{-1}
$$

From this and Theorem 3.1, we get

$$
\begin{aligned}
\chi(S) & =\left(\Pi_{5} \Pi_{4}\right) \widetilde{\Psi}(\widetilde{V})\left(\Pi_{5} \Pi_{4}\right)^{-1}=L_{S}\left(\mathbb{D}+C_{\widetilde{V}}\right)^{-1} \widetilde{\Psi}(\widetilde{V})\left(\mathbb{D}+C_{\widetilde{V}}\right) L_{S}^{-1} \\
& =L_{S}\left(\mathbb{D}-A_{\widetilde{V}}\right) \mathbb{D}^{-1}\left(\mathbb{D}+A_{\widetilde{V}}\right)\left(\mathbb{D}-C_{\widetilde{V}}\right) \mathbb{D}^{-1}\left(\mathbb{D}+C_{\widetilde{V}}\right) L_{S^{-1}} .
\end{aligned}
$$

The claim for $\chi(S)$ follows from observing that $\left(\mathbb{D}-A_{\widetilde{V}}\right) \mathbb{D}^{-1}\left(\mathbb{D}+A_{\widetilde{V}}\right)=\mathbb{D}-A_{\widetilde{V}} \mathbb{D}^{-1} A_{\widetilde{V}}=$ $\mathbb{D}-A_{N(S)} \mathbb{D}^{-1} A_{N(S)}$ and $\left(\mathbb{D}-C_{\widetilde{V}}\right) \mathbb{D}^{-1}\left(\mathbb{D}+C_{\widetilde{V}}\right)=\mathbb{D}-C_{\widetilde{V}} \mathbb{D}^{-1} C_{\widetilde{V}}=\mathbb{D}-C_{N(S)} \mathbb{D}^{-1} C_{N(S)}$. Finally,

$$
\Upsilon(\phi)=\Pi_{5}^{-1} \chi(S) \Pi_{5}=D^{-1} L_{S}\left(\mathbb{D}-A_{N(S)} \mathbb{D}^{-1} A_{N(S)}\right)\left(\mathbb{D}-C_{N(S)} \mathbb{D}^{-1} C_{N(S)}\right) L_{S}^{-1} D .
$$

Since $D L_{S}^{-1}=L_{S}^{-1} D-L_{S}^{-1} L_{S_{x}} L_{S}^{-1}$, the identity $L_{S}^{-1} D=\left(D+L_{S^{-1} S_{x}}\right) L_{S}^{-1}=\left(D+2 L_{N(S)}\right) L_{S}^{-1}=$ $\left(\mathbb{D}+A_{N(S)}\right) L_{S}^{-1}$ holds, and thus

$$
\begin{aligned}
\Upsilon(\phi) & =L_{S}\left(\mathbb{D}+A_{N(S)}\right)^{-1}\left(\mathbb{D}-A_{N(S)} \mathbb{D}^{-1} A_{N(S)}\right)\left(\mathbb{D}-C_{N(S)} \mathbb{D}^{-1} C_{N(S)}\right)\left(\mathbb{D}+A_{N(S)}\right) L_{S}^{-1} \\
& =L_{S} \mathbb{D}^{-1}\left(\mathbb{D}-A_{N(S)}\right)\left(\mathbb{D}-C_{N(S)} \mathbb{D}^{-1} C_{N(S)}\right)\left(\mathbb{D}+A_{N(S)}\right) L_{S}^{-1}
\end{aligned}
$$

which is the claim for $\Upsilon(\phi)$ upon substituting $S=\phi_{x}$.

In the scalar case, the recursion operator for the KdV sing. equation (4.2) is well-known, see, e.g., [23], where it appears in the form

$$
\Upsilon(\phi)=\phi_{x} D^{-1}\left(D^{3}+2 D \mathrm{U}+2 \mathrm{U} D\right) \phi_{x}^{-1} .
$$

with $\mathrm{U}=\frac{1}{2}\left(\phi_{x x} / \phi_{x}\right)_{x}-\frac{1}{4}\left(\phi_{x x} / \phi_{x}\right)^{2}$. To facilitate comparison to the non-Abelian case, we state the following reformulation of (4.6).

Corollary 4.8. The recursion operator $\Upsilon(\phi)$ of the $K d V$ sing. equation (4.2) in Theorem 4.7 can be written as

$$
\Upsilon(\phi)=L_{\phi_{x}} \mathbb{D}^{-1}\left(\mathbb{D}^{3}+\mathbb{D} A_{\mathrm{U}}+A_{\mathrm{U}} \mathbb{D}+C_{\mathrm{U}} \mathbb{D}^{-1} C_{\mathrm{U}}\right) L_{\phi_{x}^{-1}}
$$

where $\mathbf{U}=\left(N\left(\phi_{x}\right)\right)_{x}-\left(N\left(\phi_{x}\right)\right)^{2}$.

Proof. The corollary is an immediate consequence of Proposition 4.10, the fact that $N\left(\phi_{x}\right)=\widetilde{V}$ and $\widetilde{V}_{x}=\mathbb{D} \tilde{V}$. 
Remark 4.9. Note that in the scalar case $\mathrm{U}=v_{x}-v^{2}$, i.e., $\mathrm{U}$ stems from a Miura transformation of a solution $v$ to the $\mathrm{mKdV}$ equation. In the non-Abelian setting, we have $\mathrm{U}=\widetilde{V}_{x}-\widetilde{V}^{2}$, which means that $\mathrm{U}$ stems from a (non-Abelian) Miura transformation of a solution $\widetilde{V}$ to the amKdV equation (3.1).

Proposition 4.10. Let $\mathrm{D}$ be a derivation, and $\mathrm{U}, \mathrm{V}$ related by the Miura transform $\mathrm{U}=\mathrm{DV}-\mathrm{V}^{2}$. Then the following factorization holds:

$$
\begin{aligned}
\mathrm{D}^{3}+A_{\mathrm{U}} \mathrm{D}+\mathrm{D} A_{\mathrm{U}}+C_{\mathrm{U}} \mathrm{D}^{-1} C_{\mathrm{U}} & =\left(\mathrm{D}-A_{\mathrm{V}}\right)\left(\mathrm{D}-C_{\mathrm{V}} \mathrm{D}^{-1} C_{\mathrm{V}}\right)\left(\mathrm{D}+A_{\mathrm{V}}\right) \\
& =\left(\mathrm{D}-A_{\mathrm{V}}\right)\left(\mathrm{D} \pm C_{\mathrm{V}}\right) \mathrm{D}^{-1}\left(\mathrm{D} \mp C_{\mathrm{V}}\right)\left(\mathrm{D}+A_{\mathrm{V}}\right) .
\end{aligned}
$$

Proof. The proof of the proposition is completely analogous to the proof of the factorization of the non-Abelian KdV recursion operator on the image of a Miura transformation in [11, Proposition 14]. The crucial observation is that due to the product rule the identities

$$
\mathrm{D} A_{T}=A_{T} \mathrm{D}+A_{\mathrm{D} T}, \quad \mathrm{D} C_{T}=C_{T} \mathrm{D}+C_{\mathrm{D} T}
$$

hold for any derivation D.

There are close algebraic relations between the non-Abelian recursion operators of the amKdV, KdV sing. and int. soliton KdV equations.

Corollary 4.11. For $\widetilde{V}=\frac{1}{2} \phi_{x}^{-1} \phi_{x x}$ and $S=\phi_{x}$, the recursion operators in Theorem 3.1 and the recursion operators in (4.6), (4.7) are pairwise related. More precisely, it holds

$$
\widetilde{\Psi}(\widetilde{V})=P_{1} P_{2}=Q_{1} Q_{2}, \quad \chi(S)=Q_{2} Q_{1}=R_{1} R_{2}, \quad \Upsilon(\phi)=P_{2} P_{1}=R_{2} R_{1},
$$

where

$$
\begin{array}{lll}
P_{1}=\left(\mathbb{D}+C_{\widetilde{V}}\right)\left(\mathbb{D}+A_{\widetilde{V}}\right) L_{\phi_{x}}^{-1}, & P_{2}=L_{\phi_{x}} \mathbb{D}^{-1}\left(\mathbb{D}-A_{\widetilde{V}}\right)\left(\mathbb{D}-C_{\widetilde{V}}\right) \mathbb{D}^{-1} \\
Q_{1}=\left(\mathbb{D}+C_{\widetilde{V}}\right) L_{\phi_{x}}^{-1}, & Q_{2}=L_{\phi_{x}}\left(\mathbb{D}-A_{\widetilde{V}} \mathbb{D}^{-1} A_{\widetilde{V}}\right)\left(\mathbb{D}-C_{\widetilde{V}}\right) \mathbb{D}^{-1} \\
R_{1}=L_{\phi_{x}}\left(\mathbb{D}+A_{\widetilde{V}}\right) L_{\phi_{x}}^{-1}, & R_{2}=L_{\phi_{x}} \mathbb{D}^{-1}\left(\mathbb{D}-A_{\widetilde{V}}\right)\left(\mathbb{D}-C_{\widetilde{V}} \mathbb{D}^{-1} C_{\widetilde{V}}\right) L_{\phi_{x}}^{-1}
\end{array}
$$

\section{$5 \quad$ Hereditariness and hierarchies}

The links obtained in the last two sections are summarized in

$$
\operatorname{mKdV}(V) \stackrel{B_{2}, B_{3}}{\operatorname{amKdV}(\widetilde{V})} \frac{B_{4}}{\operatorname{KdV} \text { sing. }(\phi)} \stackrel{B_{5}}{ } \text { int. soliton } \operatorname{KdV}(S) \text {, }
$$

where the Bäcklund transformations $B_{2}, B_{3}, B_{4}$ and $B_{5}$ are given in (3.2), (3.3), (4.4) and (4.5), respectively. Applying the respective recursion operators, the Bäcklund chart extends to the whole hierarchies as follows

$$
V_{t}=[\Psi(V)]^{n} V_{x} \stackrel{B_{2}, B_{3}}{\widetilde{V}_{t}=[\widetilde{\Psi}(\widetilde{V})]^{n} \widetilde{V}_{x}} \frac{B_{4}}{\phi_{t}=[\Upsilon(\phi)]^{n} \phi_{x}} \frac{B_{5}}{S_{t}=[\chi(S)]^{n} S_{x}} .
$$

As known from the scalar case, hereditariness is a crucial property of recursion operators $[20,38]$. Unfortunately its direct verification often requires involved computations, in particular in the non-Abelian case. The following proposition uses the Bäcklund links established in the present article to avoid computations by reducing the proof of hereditariness to the hereditariness of the non-Abelian KdV recursion operator, which is proved in [54].

Proposition 5.1. Each of the recursion operators $\Psi$ from the Bäcklund chart (4.1) has the following properties: 
a) $\Psi$ is hereditary,

b) $\Psi$ is a strong symmetry for all equations of the hierarchy generated by $\Psi$.

Proof. In [54, (32)] it is verified that the non-Abelian KdV recursion operator (2.4) satisfies the identity

$$
[D, \Phi(U)]=\Phi^{\prime}(U)\left[U_{x}\right]
$$

implying that $\Phi(U)$ is a strong symmetry (recursion operator in the sense of [43]) for the trivial member $U_{t}=U_{x}$ of the non-Abelian KdV hierarchy [20]. Moreover, the main result in [54] is that $\Phi(U)$ is hereditary. Hence $\Phi(U)$ is a strong symmetry for all equations of the non-Abelian KdV hierarchy [20]. As shown in [26], the properties of a) and b) are preserved under Bäcklund transformations.

Each of the hierarchies in (5.1) is of the form

$$
U_{t}=[\Phi(U)]^{n} U_{x}
$$

We may rewrite the right-hand side of (5.2) as

$$
[\Phi(U)]^{n} U_{x}=X_{n}(U)
$$

where $X_{n}$ is a vector field on the space of $x$-dependent operator-valued functions (see [54] for details). The main consequence of Proposition 5.1 is

Corollary 5.2. Let $X_{1}, X_{2}, \ldots$ be the vector fields of one of the hierarchies in (5.2). Then we have

$$
\left[X_{m}, X_{n}\right]=0
$$

for all $m, n=1,2, \ldots$

The proof uses arguments explained in [54, Section VI].

\section{Remarks, perspectives and open problems}

This section collects some remarks on the results previously presented together with some perspectives study and open problems. The chain of Bäcklund transformations we obtained, represents a not at all trivial generalization to the operator level of the corresponding one [23] which links the scalar $\mathrm{pKdV}, \mathrm{KdV}, \mathrm{mKdV}, \mathrm{KdV}$ interacting soliton and $\mathrm{KdV}$ singularity manifold hierarchies. Furthermore, it generalizes the non-Abelian Bäcklund chart in [11] since it connects further non-Abelian hierarchies. Specifically, new non-Abelian equations, and, then, the corresponding hierarchies, arise, such as the amKdV, in (3.1).

\section{Remarks}

- The two Bäcklund charts, respectively, in the Abelian [23] (see Appendix) and the non Abelian case, connect the $\mathrm{pKdV}, \mathrm{KdV}, \mathrm{mKdV}, \mathrm{KdV}$-singularity manifold and Interacting Soliton KdV equations or their non-Abelian analogs.

- Known the recursion operator of a nonlinear evolution equation then all the other nonlinear evolution equations linked to it via a Bäcklund chart admit a recursion operator. The latter can be constructed in the Abelian as well as in the non-Abelian case. 
- All nonlinear evolution equations in the same Bäcklund chart share all the structural properties which are preserved under Bäcklund transformations as soon as a single equation, in it, enjoys them. Remarkable is the hereditariness of the recursion operators [54].

- Also in the non-Abelian operator case, given the hereditary recursion operators, the Bäcklund chart can be extended to the corresponding generated hierarchies. Again, the Bäcklund chart relates the corresponding members of each one of the involved hierarchies of nonlinear evolution equations.

- Even if there are similarities between the Abelian scalar case and the non-Abelian operator case, in the second case the structure is richer. Indeed, two distinct non-Abelian mKdV equations appear: the non-Abelian $\mathrm{mKdV}$ and an alternative $\mathrm{mKdV}$ equations which do coincide in the Abelian case. Correspondingly, when commutativity is assumed, combination of the Bäcklund transformations $B_{2}$ and $B_{3}$ produces the identity transformation and, hence, the Abelian Bäcklund chart [23] is recovered.

- A similar behavior can be observed also when the Cole-Hopf link connecting Burgers equation to linear heat equation is extended to the non-Abelian case $[12,14]$. The heat equation is connected to two different Burgers equations, termed Burgers and mirror Burgers equations in $[9,35]$. Then, recursion operators and the corresponding hierarchies follow from the Cole-Hopf link, which can be regarded as a special case of Bäcklund transformation.

\section{Perspectives and open problems}

- We expect a similar situation when the 5th order nonlinear evolution equations which appear in the Bäcklund chart in $[8,47]$ are extended to the non-Abelian case. This study is currently under investigation and we are devising also computer aided routines to check the algebraic properties of the recursion operators. Indeed, already in the Abelian case, the computations involved are very long and complicated.

- Furthermore, if a nonlinear evolution equation admits a Hamiltonian and bi-Hamiltonian structure, related to the recursion operator [21, 24, 27, 38], then all nonlinear evolution equations in the same Bäcklund chart admit a Hamiltonian and bi-Hamiltonian structure.

- The approach can be extended also when nonlinear evolution equations in $(2+1)$ dimensions are considered, namely, the unknown function is supposed to depend on two space variables rather than on a single space variable. Thus, in [40], the Kadomtsev-Petviashvili (KP), modified Kadomtsev-Petviashvili (mKP) and $(2+1)$-dimensional Harry Dym equations, which represent, in turn, the $(2+1)$-dimensional analog of $\mathrm{KdV}, \mathrm{mKdV}$, and Harry Dym equations are all connected via Bäcklund transformations. Notably, the connection among their $(1+1)$-dimensional corresponding equations [41] follows on imposing suitable constraints to the $(2+1)$-dimensional ones.

\section{A Appendix}

In this Appendix some background definitions which are of use throughout the whole article are briefly recalled in the opening subsection: this choice is due to the lack of uniqueness in many definitions in the literature. In the following subsection, some results strictly connected to the present investigation are retrieved.

\section{A.1 Background notions}

Here, to improve readability, some fundamental definitions are provided. First of all, we recall the notion of Bäcklund transformation, according to the definition of Fokas and Fuchssteiner [19] 
(see also the book by Rogers and Shadwick [49]). Consider non linear evolution equations of the type

$$
u_{t}=K(u)
$$

where the unknown function $u$ depends on the independent variables $x, t$ and, for fixed $t$, $u(x, t) \in M$, a manifold modeled on a linear topological space so that the generic fiber $T_{u} M$, at $u \in M$, can be identified with $M$ itself $^{3}$, and $K: M \rightarrow T M$, is an appropriate $C^{\infty}$ vector field on a manifold $M$ to its tangent manifold $T M$.

Let, now

$$
v_{t}=G(v)
$$

denote a second nonlinear evolution equation. Accordingly, in turn, when $u(x, t) \in M_{1}, v(x, t) \in$ $M_{2}$ and $M_{1}, M_{2}$ represent manifolds modeled on a linear topological space then, $K: M_{1} \rightarrow T M_{1}$ and $G: M_{2} \rightarrow T M_{2}$ denote appropriate $C^{\infty}$-vector fields on the manifolds $M_{i}, i=1,2$,

$$
\begin{array}{llll}
u_{t}=K(u), & K: M_{1} \rightarrow T M_{1}, & u: & (x, t) \in \mathbb{R} \times \mathbb{R} \rightarrow u(x, t) \in M_{1}, \\
v_{t}=G(v), & G: M_{2} \rightarrow T M_{2}, & v:(x, t) \in \mathbb{R} \times \mathbb{R} \rightarrow v(x, t) \in M_{2} .
\end{array}
$$

As usual, when soliton solutions are considered, the further assumption $M:=M_{1} \equiv M_{2}$ is adopted. Then, the definition of Bäcklund transformation, according to [19], can be stated as follows.

Definition A.1. Given two evolution equations, $u_{t}=K(u)$ and $v_{t}=G(v)$, then $B(u, v)=0$ represents a Bäcklund transformation between them if, whenever two solutions of these equations are given, let denote them $u(x, t)$ and $v(x, t)$ respectively, such that $B(u, v)=0$ at the initial time $t=0$, then this holds true for all times, namely,

$$
\left.B(u(x, t), v(x, t))\right|_{t=0}=\left.0 \quad \Longrightarrow \quad B(u(x, t), v(x, t))\right|_{t=\bar{t}}=0, \quad \forall \bar{t}>0, \quad \forall x \in \mathbb{R} .
$$

Therefore, the Bäcklund transformation $B$ establishes a correspondence between solutions of the evolution equations it connects. This link is graphically represented as

$$
u_{t}=K(u) \stackrel{B}{ } v_{t}=G(v)
$$

and, then, if the nonlinear evolution equation $u_{t}=K(u)$ admits a hereditary recursion operator $\Phi(u)[19]$, namely,

$$
u_{t}=\Phi(u) u_{x}, \quad \text { where } \quad K(u)=\Phi(u) u_{x},
$$

also the equation $v_{t}=G(v)$ admits a hereditary recursion operator, say $\Psi(v)$. The Bäcklund transformation $B$ allows to find $\Psi(v)$ via

$$
\Psi(v)=\Pi \Phi(u) \Pi^{-1}, \quad \text { where } \quad \Pi:=-B_{v}^{-1} B_{u}, \quad \Pi: T M_{1} \rightarrow T M_{2},
$$

where $B_{u}$ and $B_{v}$ denote the Frechet derivatives of the Bäcklund transformation $B(u, v)$. The Bäcklund transformation $B$ [19], via relation (A.3), guarantees that $\Psi(v)$ represents the hereditary recursion operator admitted by the equation $v_{t}=G(v)$. Hence, the two hierarchies $u_{t}=$ $[\Phi(u)]^{n} u_{x}$ and $v_{t}=[\Psi(v)]^{n} v_{x}, n \geq 0$, of nonlinear evolution equations can be constructed [20]

\footnotetext{
${ }^{3}$ It is generally assumed that $M$ is the space of functions $u(x, t)$ which, for each fixed $t$, belong to the Schwartz space $S$ of rapidly decreasing functions on $\mathbb{R}^{n}$, i.e., $S\left(\mathbb{R}^{n}\right):=\left\{f \in C^{\infty}\left(\mathbb{R}^{n}\right):\|f\|_{\alpha, \beta}<\infty, \forall \alpha, \beta\right\}$, where $\|f\|_{\alpha, \beta}:=$ $\sup _{x \in \mathbb{R}^{n}}\left|x^{\alpha} D^{\beta} f(x)\right|$, and $D^{\beta}:=\partial^{\beta} / \partial x^{\beta}$. In the present section $n=1$.
} 
and their base members equations are, in turn, (A.1) and (A.2). Each equation in these two hierarchies, parametrized ${ }^{4}$ by $n \in \mathbb{N}_{0}$, is connected, via the same Bäcklund transformation $B$, to the corresponding equation, parametrized by the same $n$, in the other hierarchy. This extension to the whole hierarchies is depicted in the following Bäcklund chart

$$
u=[\Phi(u)]^{n} u_{x} \stackrel{B}{-} v_{t}=[\Psi(v)]^{n} v_{x},
$$

which emphasizes that the link between the two equations (A.1) and (A.2) is inherited ${ }^{5}$ by each member of the two hierarchies generated, respectively, by the recursion operators $\Phi$ and $\Psi$.

\section{A.2 Abelian KdV connected Bäcklund chart}

Here some results, obtained in [23], concerning the links among nonlinear evolution equations whose base member is a 3-rd order equation, are recalled. An overview on further previous result is given in [7]. Thus, the Bäcklund chart here below, (A.4), allows to find solutions of Harry Dym equation [28]. This method to find solutions is not restricted to third order nonlinear evolution equations; indeed, for instance, also solutions of Burgers equation are obtained via Bäcklund transformations (see $[17,30,33,36]$ ) to mention a few of them.

The following Bäcklund chart summarizes links relating 3rd order nonlinear evolution equations, when the links in [23] are combined with the link between the KdV and potential KdV $(\mathrm{pKdV})$,

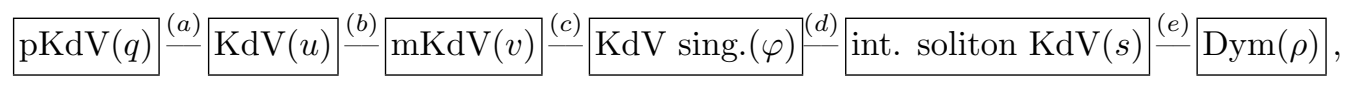

wherein the third order nonlinear evolution equations are, in turn, given by

$$
\begin{aligned}
& q_{t}=q_{x x x}+3 q_{x}^{2} \quad \quad(\mathrm{pKdV}), \\
& u_{t}=u_{x x x}+6 u u_{x} \quad(\mathrm{KdV}), \\
& v_{t}=v_{x x x}-6 v^{2} v_{x} \quad(\mathrm{mKdV}), \\
& \varphi_{t}=\varphi_{x}\{\varphi ; x\} \text {, where }\{\varphi ; x\}:=\left(\frac{\varphi_{x x}}{\varphi_{x}}\right)_{x}-\frac{1}{2}\left(\frac{\varphi_{x x}}{\varphi_{x}}\right)^{2} \quad \text { (KdV sing.), } \\
& s^{2} s_{t}=s^{2} s_{x x x}-3 s s_{x} s_{x x}+\frac{3}{2} s_{x}^{3} \quad \text { (int. soliton KdV), }
\end{aligned}
$$

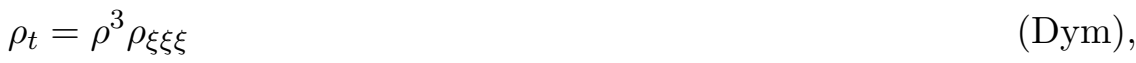

in addition, the Bäcklund transformations which link them, are, respectively:
(a) $u-q_{x}=0$,
(b) $u+v_{x}+v^{2}=0$
(c) $v-\frac{1}{2} \frac{\varphi_{x x}}{\varphi_{x}}=0$
(d) $s-\varphi_{x}=0$
(e) $\bar{x}:=D^{-1} s(x)$
where $D^{-1}:=\int_{-\infty}^{x} d \xi$

The transformation (e) is a reciprocal ${ }^{6}$ transformation [4, 49]: according to [8, 23], it represents a Bäcklund transformation whose transformation operator $\Pi$ can be constructed, provided it is

\footnotetext{
${ }^{4}$ Here and throught $\mathbb{N}_{0}:=\mathbb{N} \cup\{0\}$.

${ }^{5}$ These recursion operators are termed hereditary in [20] to stress that the property to be recursion operator, established referring to the base member equation, is inherited by all the other members in the hierarchy of nonlinear evolution equations.

${ }^{6}$ Reciprocal-type transformations were originally introduced in $(2+0)$-dimensional isentropic gasdynamics by Bateman [3, 4] and in $(1+1)$-dimensional anisentropic gasdynamics by Rogers [45]. Connection with invariance of these gasdynamic systems under Bäcklund transformations is described in [49].
} 
considered an extended manifold which comprises also the independent space variable $x$, further to the dependent variable $u$. That is, while the Bäcklund transformations $(a),(b)$ and $(c)$ admit operators $\Pi$

$$
\Pi_{a}: T M_{u} \rightarrow T M_{v}, \quad \Pi_{b}: T M_{v} \rightarrow T M_{\varphi}, \quad \Pi_{c}: T M_{\varphi} \rightarrow T M_{s}
$$

when the reciprocal transformation $(e)$ is considered, the corresponding operator is

$$
\Pi_{e}: T M_{(x, s)} \rightarrow T M_{(\bar{x}, \rho)}
$$

The Bäcklund chart (A.4) allows to find solutions to initial boundary value problems of the Dym equation $[23,28]$. Furthermore, the links depicted in the Bäcklund chart relate each member of the involved hierarchies to the corresponding member of all the other hierarchies in the Bäcklund chart. Composition of Bäcklund transformations in (A.4) allows to retrieve and generalize to the whole hierarchies, the direct Bäcklund transformation between the KdV and Dym equations in [31].

The Bäcklund chart (A.4) turned out to be very helpful in revealing new invariances enjoyed by any of the equations which appear in the Bäcklund chart itself. Indeed, let

$$
\{\varphi ; x\}=\left(\frac{\varphi_{x x}}{\varphi_{x}}\right)_{x}-\frac{1}{2}\left(\frac{\varphi_{x x}}{\varphi_{x}}\right)^{2}
$$

denote the Schwarzian derivative, then, the KdV Singularity equation $\varphi_{t}=\varphi_{x}\{\varphi ; x\}$ is invariant under the Möbius group of transformations

$$
\widetilde{\varphi}=\frac{a \varphi+b}{c \varphi+d}, \quad a, b, c, d \in \mathbb{C} \quad \text { such that } \quad a d-b c \neq 0,
$$

since the Schwarzian derivative of $\varphi$ with respect to $x,\{\varphi ; x\}$, enjoys such a property.

The invariance, under the Möbius group of transformations of all the members of the KdV Singularity hierarchy allowed to recover the Invariance I, enjoyed by the Dym hierarchy [23] and to find a new invariance enjoyed by the Kawamoto equation [47] in the case of the 5th order Bäcklund chart.

\section{Acknowledgements}

The financial support of G.N.F.M.-I.N.d.A.M., I.N.F.N. and Sapienza University of Rome, Italy are gratefully acknowledged. C. Schiebold wishes also to thank S.B.A.I. Dept. and Sapienza University of Rome for the kind hospitality. The authors wish to thank the referees who carefully read this work: their comments were of help in improving its presentation.

\section{References}

[1] Aden H., Carl B., On realizations of solutions of the KdV equation by determinants on operator ideals, J. Math. Phys. 37 (1996), 1833-1857.

[2] Athorne C., Fordy A., Generalised KdV and MKdV equations associated with symmetric spaces, J. Phys. A: Math. Gen. 20 (1987), 1377-1386.

[3] Bateman H., The lift and drag functions for an elastic fluid in two-dimensional irrotational flow, Proc. Nat. Acad. Sci. USA 24 (1938), 246-251.

[4] Bateman H., The transformation of partial differential equations, Quart. Appl. Math. 1 (1944), $281-296$.

[5] Calogero F., Degasperis A., Nonlinear evolution equations solvable by the inverse spectral transform. II, Nuovo Cimento B 39 (1977), 1-54. 
[6] Calogero F., Degasperis A., Spectral transform and solitons. Vol. I. Tools to solve and investigate nonlinear evolution equations, Studies in Mathematics and its Applications, Vol. 13, North-Holland Publishing Co., Amsterdam - New York, 1982.

[7] Carillo S., Nonlinear evolution equations: Bäcklund transformations and Bäcklund charts, Acta Appl. Math. 122 (2012), 93-106.

[8] Carillo S., Fuchssteiner B., The abundant symmetry structure of hierarchies of nonlinear equations obtained by reciprocal links, J. Math. Phys. 30 (1989), 1606-1613.

[9] Carillo S., Lo Schiavo M., Schiebold C., Recursion operators admitted by non-Abelian Burgers equations: some remarks, arXiv:1606.07270.

[10] Carillo S., Rogers C., Bäcklund charts for the Caudrey-Dodd-Gibbon and Kaup-Kupershmidt hierarchies, in Nonlinear Evolutions (Balaruc-les-Bains, 1987), World Sci. Publ., Teaneck, NJ, 1988, 57-73.

[11] Carillo S., Schiebold C., Noncommutative Korteweg-de Vries and modified Korteweg-de Vries hierarchies via recursion methods, J. Math. Phys. 50 (2009), 073510, 14 pages.

[12] Carillo S., Schiebold C., A non-commutative operator-hierarchy of Burgers equations and Bäcklund transformations, in Applied and Industrial Mathematics in Italy III, Ser. Adv. Math. Appl. Sci., Vol. 82, World Sci. Publ., Hackensack, NJ, 2010, 175-185.

[13] Carillo S., Schiebold C., Matrix Korteweg-de Vries and modified Korteweg-de Vries hierarchies: noncommutative soliton solutions, J. Math. Phys. 52 (2011), 053507, 21 pages.

[14] Carillo S., Schiebold C., On the recursion operator for the noncommutative Burgers hierarchy, J. Nonlinear Math. Phys. 19 (2012), 1250003, 11 pages.

[15] Carl B., Schiebold C., Nonlinear equations in soliton physics and operator ideals, Nonlinearity 12 (1999), 333-364.

[16] Carl B., Schiebold C., Ein direkter Ansatz zur Untersuchung von Solitonengleichungen, Jahresber. Deutsch. Math.-Verein. 102 (2000), 102-148.

[17] Cole J.D., On a quasi-linear parabolic equation occurring in aerodynamics, Quart. Appl. Math. 9 (1951), 225-236.

[18] Depireux D.A., Schiff J., On UrKdV and UrKP, Lett. Math. Phys. 33 (1995), 99-111, solv-int/9402004.

[19] Fokas A.S., Fuchssteiner B., Bäcklund transformations for hereditary symmetries, Nonlinear Anal. 5 (1981), 423-432.

[20] Fuchssteiner B., Application of hereditary symmetries to nonlinear evolution equations, Nonlinear Anal. 3 (1979), 849-862.

[21] Fuchssteiner B., The Lie algebra structure of degenerate Hamiltonian and bi-Hamiltonian systems, Progr. Theoret. Phys. 68 (1982), 1082-1104.

[22] Fuchssteiner B., Solitons in interaction, Progr. Theoret. Phys. 78 (1987), 1022-1050.

[23] Fuchssteiner B., Carillo S., Soliton structure versus singularity analysis: third-order completely integrable nonlinear differential equations in 1+1-dimensions, Phys. A 154 (1989), 467-510.

[24] Fuchssteiner B., Carillo S., The action-angle transformation for soliton equations, Phys. A 166 (1990), 651-675.

[25] Fuchssteiner B., Chowdhury A.R., A new approach to the quantum KdV, Chaos Solitons Fractals 5 (1995), 2345-2355.

[26] Fuchssteiner B., Fokas A.S., Symplectic structures, their Bäcklund transformations and hereditary symmetries, Phys. D 4 (1981), 47-66.

[27] Fuchssteiner B., Oevel W., The bi-Hamiltonian structure of some nonlinear fifth- and seventh-order differential equations and recursion formulas for their symmetries and conserved covaria, J. Math. Phys. 23 (1982), 358-363.

[28] Fuchssteiner B., Schulze T., Carillo S., Explicit solutions for the Harry Dym equation, J. Phys. A: Math. Gen. 25 (1992), 223-230.

[29] Gu C., Hu H., Zhou Z., Darboux transformations in integrable systems. Theory and their applications to geometry, Mathematical Physics Studies, Vol. 26, Springer, Dordrecht, 2005.

[30] Guo B.Y., Carillo S., Infiltration in soils with prescribed boundary concentration, Acta Math. Appl. Sinica 6 (1990), 365-369.

[31] Guo B.Y., Rogers C., On Harry-Dym equation and its solution, Sci. China Ser. A 32 (1989), $283-295$. 
[32] Gürses M., Karasu A., Sokolov V.V., On construction of recursion operators from Lax representation, J. Math. Phys. 40 (1999), 6473-6490, solv-int/9909003.

[33] Hopf E., The partial differential equation $u_{t}+u u_{x}=\mu u_{x x}$, Comm. Pure Appl. Math. 3 (1950), 201-230.

[34] Khalilov F.A., Khruslov E.Ya., Matrix generalisation of the modified Korteweg-de Vries equation, Inverse Problems 6 (1990), 193-204.

[35] Kupershmidt B.A., On a group of automorphisms of the noncommutative Burgers hierarchy, J. Nonlinear Math. Phys. 12 (2005), 539-549.

[36] Levi D., Ragnisco O., Bruschi M., Continuous and discrete matrix Burgers' hierarchies, Nuovo Cimento B 74 (1983), 33-51.

[37] Liu Q.P., Athorne C., Comment on "Matrix generalisation of the modified Korteweg-de Vries equation", Inverse Problems 7 (1991), 783-785.

[38] Magri F., A simple model of the integrable Hamiltonian equation, J. Math. Phys. 19 (1978), 1156-1162.

[39] Marchenko V.A., Nonlinear equations and operator algebras, Mathematics and its Applications (Soviet Series), Vol. 17, D. Reidel Publishing Co., Dordrecht, 1988.

[40] Oevel W., Carillo S., Squared eigenfunction symmetries for soliton equations. I, J. Math. Anal. Appl. 217 (1998), 161-178.

[41] Oevel W., Carillo S., Squared eigenfunction symmetries for soliton equations. II, J. Math. Anal. Appl. 217 (1998), 179-199.

[42] Oevel W., Rogers C., Gauge transformations and reciprocal links in $2+1$ dimensions, Rev. Math. Phys. 5 (1993), 299-330.

[43] Olver P.J., Evolution equations possessing infinitely many symmetries, J. Math. Phys. 18 (1977), 1212-1215.

[44] Olver P.J., Sokolov V.V., Integrable evolution equations on associative algebras, Comm. Math. Phys. 193 (1998), 245-268.

[45] Rogers C., Reciprocal relations in non-steady one-dimensional gasdynamics, Z. Angew. Math. Phys. 19 (1968), 58-63.

[46] Rogers C., Ames W.F., Nonlinear boundary value problems in science and engineering, Mathematics in Science and Engineering, Vol. 183, Academic Press, Inc., Boston, MA, 1989.

[47] Rogers C., Carillo S., On reciprocal properties of the Caudrey-Dodd-Gibbon and Kaup-Kupershmidt hierarchies, Phys. Scripta 36 (1987), 865-869.

[48] Rogers C., Schief W.K., Bäcklund and Darboux transformations. Geometry and modern applications in soliton theory, Cambridge Texts in Applied Mathematics, Cambridge University Press, Cambridge, 2002.

[49] Rogers C., Shadwick W.F., Bäcklund transformations and their applications, Mathematics in Science and Engineering, Vol. 161, Academic Press, Inc., New York - London, 1982.

[50] Schiebold C., From the non-abelian to the scalar two-dimensional Toda lattice, Glasg. Math. J. 47 (2005), 177-189.

[51] Schiebold C., Noncommutative AKNS systems and multisoliton solutions to the matrix sine-Gordon equation, Discrete Contin. Dyn. Syst. 2009 (2009), 678-690.

[52] Schiebold C., Cauchy-type determinants and integrable systems, Linear Algebra Appl. 433 (2010), $447-475$.

[53] Schiebold C., The noncommutative AKNS system: projection to matrix systems, countable superposition and soliton-like solutions, J. Phys. A: Math. Theor. 43 (2010), 434030, 18 pages.

[54] Schiebold C., Structural properties of the noncommutative KdV recursion operator, J. Math. Phys. 52 (2011), 113504, 16 pages.

[55] Schiff J., Symmetries of KdV and loop groups, solv-int/9606004.

[56] Svinolupov S.I., Sokolov V.V., Vector-matrix generalizations of classical integrable equations, Theoret. and Math. Phys. 100 (1994), 959-962.

[57] Weiss J., On classes of integrable systems and the Painlevé property, J. Math. Phys. 25 (1984), $13-24$.

[58] Wilson G., On the quasi-Hamiltonian formalism of the KdV equation, Phys. Lett. A 132 (1988), 445-450. 